\title{
JAK/STAT signaling pathway-mediated microRNA-181b promoted blood-brain barrier impairment by targeting sphingosine-1-phosphate receptor 1 in septic rats
}

\author{
Sheng-Long Chen ${ }^{1 \#}$, Geng-Xin Cai ${ }^{2 \#}$, Hong-Guang Ding ${ }^{1 \#}$, Xin-Qiang Liu ${ }^{1}$, Zhong-Hua Wang ${ }^{1}$, \\ Yuan-Wen Jing ${ }^{1}$, Yong-Li Han ${ }^{1}$, Wen-Qiang Jiang ${ }^{1}$, Miao-Yun Wen ${ }^{1,2,3}$ \\ ${ }^{1}$ Department of Critical Care Medicine, Guangdong Provincial People's Hospital, Guangdong Academy of Medical Sciences, Guangzhou, China; \\ ${ }^{2}$ South China University of Technology School of Medicine, Guangzhou, China; ${ }^{3}$ Southern Medical University, Guangzhou, China \\ Contributions: (I) Conception and design: SL Chen, GX Cai, MY Wen; (II) Administrative support: WQ Jiang; (III) Provision of study materials \\ or patients: XQ Liu, ZH Wang, YW Jing; (IV) Collection and assembly of data: SL Chen, GX Cai, HG Ding, XQ Liu; (V) Data analysis and \\ interpretation: YL Han, WQ Jiang, MY Wen; (VI) Manuscript writing: All authors; (VII) Final approval of manuscript: All authors. \\ \#These authors contributed equally to this work. \\ Correspondence to: Wen-Qiang Jiang, PhD, MD; Miao-Yun Wen, PhD, MD. 106 Zhongshan Er Road, Guangzhou 510080, China. \\ Email: wenjiang8046@163.com; wenmiaoyun041105@163.com.
}

Background: Blood-brain barrier (BBB) impairment plays a significant role in the pathogenesis of sepsisassociated encephalopathy (SAE). However, the molecular mechanisms are poorly understood. In the present study, we aimed to investigate the regulatory relationship between the Janus kinase/signal transducers and activators of transcription (JAK/STAT) signaling pathway, microRNA (miR)-181b and its target genes in sepsis in vivo and in vitro.

Methods: Four rat models (sham, sepsis, sepsis plus STAT3 inhibitor (Stattic), and sepsis plus miR-181b inhibitor [sepsis + anta-miR-181b]) were established. For the in vitro experiments, rat brain microvascular endothelial cells (rBMECs) and rat brain astrocytes (rAstrocytes) were cultured with 10\% serum harvested from sham, sepsis, and sepsis + anta-miR-181b rats. Chromatin immunoprecipitation-quantitative polymerase chain reaction (ChIP-QPCR) analysis was carried out to detect the binding and enrichment of the JAK/STAT3 signal core transcription complex in the miR-181b promoter region. Dual-luciferase reporter gene assay was conducted to test miR-181b and its target genes. The cell adhesion rate of rBMECs was also measured.

Results: During our investigations, the expression levels of miR-181b, p-JAK2, p-STAT3, and C/EBP $\beta$ were found to be significantly increased in the septic rats compared with the sham rats. STAT3 inhibitor halted BBB damage by downregulating the expression of miR-181b. In addition, miR-181b targeted sphingosine-1-phosphate receptor 1 (S1PR1) and neurocalcin delta (NCALD). The up-regulated miR-181b significantly decreased the cell adhesion rate of rBMECs. The administration of miR-181b inhibitor reduced damage to the BBB through increasing the expression of S1PR1 and NCALD, which again proved that miR181 b negatively regulates SIPR1 and NCALD to induce BBB damage.

Conclusions: Our study demonstrated that JAK2/STAT3 signaling pathway induced expression of miR$181 \mathrm{~b}$, which promoted BBB impairment in rats with sepsis by downregulating S1PR1 and decreasing BBB cell adhesion. These findings strongly suggest JAK2/STAT3/miR-181b axis as therapeutic target in protecting against sepsis-induced BBB damage.

Keywords: Sepsis; blood-brain barrier (BBB); microRNA-181b (miR-181b); JAK/STAT signaling pathway; sphingosine-1-phosphate receptor 1 (S1PR1)

Submitted Sep 27, 2020. Accepted for publication Nov 10, 2020.

doi: $10.21037 / \mathrm{atm}-20-7024$

View this article at: http://dx.doi.org/10.21037/atm-20-7024 


\section{Introduction}

Sepsis is a common complication following severe infection, severe trauma, burns, shock, and surgery. It occurs when the body's reaction to an infection causes damage to its own tissues and organs. Sepsis can lead to severe sepsis, which involves organ failure and can further progress onto septic shock, multiple organ dysfunction syndrome (MODS), and life-threatening low blood pressure. A meta-analysis in 2016 estimated the annual number of sepsis cases diagnosed globally to be 31.5 million, with more than half being severe sepsis cases (1) The hospital mortality rate for sepsis and severe sepsis was found to be $17 \%$ and $26 \%$, respectively (1). A high percentage of sepsis survivors experience physical and psychological sequela, including cognitive dysfunction, also known as sepsis-associated encephalopathy (SAE) (2).

Some studies have demonstrated that injury to the blood-brain barrier (BBB) plays a key role in the pathophysiological mechanism of SAE $(3,4)$. Annane and Sharshar (3) demonstrated that interventions targeting the blood-brain barrier have shown promise in prevention of cognitive dysfunction in various experimental models of sepsis. Moreover, the BBB protects brain tissue from being penetrated by harmful substances. The barrier between blood plasma and brain cells is maintained by the brain capillary walls and glial cells, whereas the barrier between plasma and cerebrospinal fluid is maintained by the choroid plexus (5). BBB maintains the stability of the internal environment and is thus essential for maintaining the normal biological and physiological state of the central nervous system. Therefore, the destruction of the BBB induces loss of tissue metabolite balance, infiltration of peripheral macrophages and lymphocytes, cerebral edema, promotion of inflammatory responses, and inhibition of nerve repair, thereby damaging brain function and causing SAE. Sepsis-induced damage to the brain first manifests as the destruction of cerebral capillaries, which promotes the activation of glial cells, triggering a series of proinflammatory cytokines in the central nervous system, and eventually leading to brain dysfunction (6). It is therefore clear that an impaired BBB can lead to SAE, which severely affects the function of the central nervous system. However, the molecular mechanisms by which BBB impairment leads to SAE in sepsis survivors have been rarely studied.

The Janus kinase/signal transducers and activators of transcription (JAK/STAT) signaling pathway has been shown related to the release of various cytokines and inflammatory mediators and involved in the regulation of immune response in sepsis $(7,8)$. Moreover, JAK/STAT pathway has been shown involved in organ damage (heart and lungs) and other dysfunctions in sepsis models (9-11). Chang et al. (12) demonstrated that the JAK/STAT signaling pathway was involved in the occurrence of sepsis and regulation of inflammation by significantly upregulated expression of protein and mRNA expression of JAK and STAT3, and increased transcription and expression of inflammatory factors. JAK/STAT signaling pathway has also been shown to regulate the levels of serum factors used in the diagnosis and prognosis of sepsis patients (13). Moreover, miRNA-30a and miR-155 were reported to relieve sepsis-induced liver injury through inactivating JAK/ STAT pathway $(14,15)$. Considering that inflammatory cytokines were released by JAK/STAT signaling pathway and was harmful for BBB, we wondered whether JAK/ STAT signaling pathway regulates the BBB or further influences SAE, and if so, how. Besides, none of the studies have revealed the relationship in septic rat before.

As a member of the signal transduction and transcription activator protein family, STAT3 has the unique property of binding DNA (16). It polymerizes into an activated transcription activator in a homologous or heterodimer manner prior to phosphorylation. Phosphorylated STAT3 (p-STAT3) then bursts into the nucleus, binds to a specific site in the promoter sequence, and promotes transcription of cytokines and inflammatory cytokines (17). In addition to being a transcriptional activator of some inflammatory factor genes, STAT3 can also transcriptionally regulate noncoding RNAs, including microRNAs (miRNAs) $(18,19)$.

The miR-181 family, which includes miR-181a, miR$181 \mathrm{~b}, \mathrm{miR}-181 \mathrm{c}$, and miR-181d, has been found to target the three prime untranslated region (3'-UTR) sequence of inflammatory factors (20). Moreover, miR-181b inhibits the expression of pro-inflammatory genes induced by TNF- $\alpha$ in human umbilical vein endothelial cells (21). Studies have confirmed that STAT3 and C/EBP $\beta$ jointly regulate the abnormal expression of miR-181b in septic rats, with miR-181b promoting the inhibitory effect of bone marrow-derived suppressor cells on immune function in sepsis and ultimately promoting death in the rats $(22,23)$. Moreover, S100A9 was supposed to promote MDSC expansion and immunosuppression in late/chronic sepsis by inducing the expression of miR-181b (24). Not even that, studies also illustrated the role of miR-181b on regulating proinflammatory factors, which showed that miR-181b$5 \mathrm{p}$ inhibitor induced upregulation of proinflammatory factors $(21,25)$. Taken the role of miR-181b and JAK-STAT 
signaling pathway on inflammatory into consideration, we wonder whether the miRNA-181b also play an important role in blood-brain barrier sepsis-induced brain injury. Therefore, we speculate that if $\mathrm{miR}-181 \mathrm{~b}$ can regulate function mechanism of BBB, rather than just affect inflammatory processes of the central nervous system.

The present study was conducted to investigate the role of the JAK-STAT3 signaling pathway and the regulation of miR-181b by STAT3 and C/EBP $\beta$ in sepsis-induced BBB damage. The association between JAK/STAT3 and sepsisinduced BBB injury was validated through studying JAK/ STAT3 activation and miR-181b expression in rat models of sepsis. The effects of JAK/STAT3 and miR-181b on brain injury of sepsis rat model were then assessed using a STAT3 inhibitor and a miR-181b antagonist. Finally, the potential target genes and mechanisms of miR-181b in sepsis were explored. We present the following article in accordance with the ARRIVE reporting checklist (available at http:// dx.doi.org/10.21037/atm-20-7024).

\section{Methods}

\section{Experimental animals}

Forty specific-pathogen-free (SPF) male Sprague-Dawley (SD) rats (8 weeks old, weight: $200-250 \mathrm{~g}$ ) were obtained from the Laboratory Animal Center of Sun Yat-sen University. The rats were randomly assigned into four groups: the sham-operation group, sepsis model group, sepsis model injected with STAT3 inhibitor (Stattic) group, and sepsis model injected with miR-181b inhibitor (sepsis + anta-miR-181b) group. The rats were housed in separate cages at adaptive feeding periods under room temperatures of $21-25^{\circ} \mathrm{C}$ with $12 \mathrm{~h}: 12 \mathrm{~h}$ light/dark cycle and free access to food and water.

The reared SD rats were fasted for 12 hours before the operation with free access to drinking water. Rats were then anesthetized by intraperitoneal injection of $5 \%$ chloral hydrate $(0.6 \mathrm{~mL} / 100 \mathrm{~g}$ body weight). After confirmation of anesthesia, the rats were fixed and routinely disinfected. The abdomen was depilated, and a $1.5-\mathrm{cm}$ incision was performed along the midline of the abdomen. The mesentery around the cecum was freed while avoiding damage to the mesangial blood vessels. In the sham operation group, the cecum was returned to the abdominal cavity and the abdomen was closed layer by layer. In the sepsis group, the cecum was ligated with No. 4 surgical sutures at one-third of the distance from the cecum root and the cecum was pierced with a 3 -mm-wide cone at approximately $0.8-1 \mathrm{~cm}$ from the distal end of the ligation. After the operation, the cecum was placed back into the abdominal cavity and the abdomen was closed layer by layer. The rats in the Stattic group and the sepsis + antamiR-181b group were intravenously injected with Stattic $(20 \mathrm{mg} / \mathrm{kg})$ or mi-181b antagomir ( $2 \mathrm{nmol})$, respectively, 12 $\mathrm{h}$ before the operation. Then, both the Stattic and sepsis + anta-miR-181b models were constructed in the same way as the sepsis model. After the model construction, the rats were routinely disinfected, reared separately, and kept under close observation. Experiments were performed under a project license (NO.: EC2020-0415) granted by institutional ethics committee board of Guangdong Academy of Medical Sciences, in compliance with the National Institutes of Health Guide concerning the Care and Use of Laboratory Animals.

\section{Cell culture and apoptosis assessment}

Rat brain microvascular endothelial cells (rBMECs) and rat brain astrocytes (rAstrocytes) were purchased from iCell Bioscience Inc (Shanghai, China) and cultured at $37^{\circ} \mathrm{C}$ with $5 \%$ carbon dioxide $\left(\mathrm{CO}_{2}\right)$. Cells were cultured with $10 \%$ serum harvested from the sham, sepsis, and sepsis + antamiR-181b rats for $24 \mathrm{~h}$. Therefore, the in vitro cell groups were: (1 and 2) rBMECs and rAstrocytes treated with serum from sham rats; (3 and 4) rBMECs and rAstrocytes treated with serum from septic rats; and (5 and 6) rBMECs and rAstrocytes treated with serum from sepsis + miR-181b inhibitor rats. Each experiment was repeated three times. After $24 \mathrm{~h}$ of induction, cells were collected for quantitative real-time polymerase chain reaction (qRT-PCR) and western blot analysis.

\section{Hoechst staining}

Hoechst 33258 staining was purchased from Beyotime (Shanghai, China). Cultured cells from the six groups mentioned above were stained in the dark with Hoechst 33258 at $25^{\circ} \mathrm{C}$ for $15 \mathrm{~min}$ and observed under a fluorescence microscope.

\section{Immunobistochemistry and terminal deoxynucleotidyl transferase dUTP nick end labeling (TUNEL) staining}

Immunohistochemistry was performed on rBMECs and rAstrocytes treated with $10 \%$ serum collected from sham, 
sepsis, and sepsis + anta-miR-181b rats. All sections of brains were immersed into $4 \%$ paraformaldehyde for 20 min, and then washed three times with phosphate-buffered saline (PBS; 5 min each time). Next, they were immersed in $0.5 \%$ triton for $20 \mathrm{~min}$, and washed three times with PBS (5 min each time). The sections were incubated with $10 \%$ goat serum for $30 \mathrm{~min}$, followed by incubation overnight at $4{ }^{\circ} \mathrm{C}$ with primary antibodies of JAK2 p-Y1007 + Y1008 (1:1,000; ab32101, Abcam), STAT3 p-Y705 (1:500; ab76315), sphingosine-1-phosphate receptor 1 (S1PR1; 1:500; ab11424), and neurocalcin delta (NCALD; 1:1,000; PA5-76322, Invitrogen). Following that, the sections were washed three times with PBS and incubated with the secondary antibody at $37^{\circ} \mathrm{C}$ for $30 \mathrm{~min}$. After washing with PBS three times, the sections were deparaffinized using anti-fluorescence quenching mounting tablets containing 2-(4-Amidinophenyl)-6-indolecarbamidine dihydrochloride (DAPI), protected from light at $4{ }^{\circ} \mathrm{C}$, and observed using a confocal laser microscope (Leica, Weztlar, Germany).

Cell apoptosis was evaluated using a terminal transferasemediated UTP nick end-labeling (TUNEL) kit (In Situ Cell Death Detection Kit, Roche, Germany) according to the manufacturer's instructions. For each section, five randomly selected visual fields were evaluated using a highpower lens. Confocal laser microscopy was used to obtain images from DAPI and TUNEL analyses.

\section{Evans blue dye (EBD) extravasation}

The destruction of the BBB can cause increased permeability of capillaries in the brain tissue. Albumin bound to $\mathrm{EBD}$ can enter the brain tissue via the $\mathrm{BBB}$, and its exudation in the brain tissue can be measured using a chemical colorimetric method, which can reflect damage to the BBB. In the present study, EBD extravasation was used to assess permeability in sham, sepsis, Stattic, and sepsis + miR-181b inhibitor (sepsis + anta-miR-181b) rats. Three rats in each group were injected with EBD solution before the operation, and photos were taken of the EBD-stained brain tissue.

\section{Enzyme-linked immunosorbent assay analysis of pro- inflammatory cytokines}

Sepsis triggers the release of numerous pro-inflammatory cytokines, including tumor necrosis factor-alpha (TNF- $\alpha$ ) and interleukins (ILs) such as IL-6 and IL-8, which have become indicators of the sepsis rat model. Serum from sham, sepsis, Stattic, and sepsis + anta-miR-181b rats was extracted and used for the detection of inflammatory factors, including D-lactic acid, TNF- $\alpha$, IL-6, and IL8. D-lactate, TNF- $\alpha$, IL-6, and IL-8 detection kits were purchased from Jiangsu Enzyme Immunoassay Industry Co., Ltd (Yencheng, China). All rats in the four groups were anesthetized and blood was collected. To extract the serum, whole blood samples were placed at room temperature for $30 \mathrm{~min}$ to 2 hours without vigorous shaking. The samples were then centrifuged at $1,000-2,000 \mathrm{~g}$ at $4{ }^{\circ} \mathrm{C}$ for $10 \mathrm{~min}$, and the yellow supernatant was collected.

The concentrations of D-lactate, TNF- $\alpha$, IL- 6 , and IL-8 in the brain tissue of sham, sepsis, Stattic, and sepsis + anta-miR-181b were also measured. Approximately 0.2$1 \mathrm{~g}$ of brain tissue was taken from fixed parts of rat brains and washed in ice-cold normal saline at $4{ }^{\circ} \mathrm{C}$. The tissues were then cut into pieces in $0.86 \%$ ice-cold normal saline, where the volume of saline was nine times more than the tissue volume. The tissue was then homogenized using a glass homogenization tube. Finally, the prepared $10 \%$ homogenate was centrifuged in an ordinary centrifuge or a low-temperature low-speed centrifuge at 3,000 rpm for 10-15 min. The supernatant was collected and stored at $4{ }^{\circ} \mathrm{C}$. The concentrations of D-lactic acid, TNF- $\alpha$, IL- 6 , and IL- 8 in the rat serum and brain tissue were measured according to the manufacturer's protocol.

\section{Western blot analysis}

The protein expression levels of C/EBP $\beta$ and other proteins involved in the JAK-STAT3 signaling pathway, such as JAK2, phosphorylated JAK2 (p-JAK2), STAT3, and phosphorylated STAT3 (p-STAT3) (p-STAT3), were evaluated using western blot, with glyceraldehyde3 -phosphate dehydrogenase (GAPDH) protein as the reference. PierceTM BCA Protein Assay Kit (no. 23227; Thermo Scientific, USA) was used to evaluate the protein concentration of samples.

Sodium dodecyl sulphate-polyacrylamide gel electrophoresis (SDS-PAGE) was performed according to standard procedures using the Bio-Rad Protean II minigel system (Bio-Rad, Hercules, USA). A total of $20 \mu \mathrm{g}$ protein of each sample was electrophoretically separated in $10 \%$ sodium dodecyl sulfate separation gel under $100 \mathrm{~V}$ and concentration gel under $120 \mathrm{~V}$ until the bromophenol blue reached to bottom. The proteins on the gel were then transferred onto a polyvinylidene fluoride (PVDF) membrane. The PVDF membrane was washed with $25 \mathrm{~mL}$ 
Table 1 The primer sequences of real-time quantitative polymerase chain-reaction (RT-qPCR)

\begin{tabular}{ll}
\hline Gene & Sequence (5'-3') \\
\hline F6 & F' 5'-CTCGCTTCGGCAGCACA-3' \\
GAPDH & F: 5'-CCTCGTCTCATAGACAAGATGGT-3' \\
& R: 5'-GGGTAGAGTCATACTGGAACATG-3' \\
S1PR1 & F: 5'-AGCTAACCTGCTGTTGTCTG-3' \\
& R: 5'-CTCAATGGCGATAGCAAGGA-3' \\
NCALD & R' 5'-GCACAGACTTCACAGAGCAT-3' \\
& RT: CTCAACTGGTGTCGTGGAGTCGGCAATTCAGTTGAGACCCACCG \\
rno-miR-181b-5p & R: ACACTCCAGCTGGGACATTCATTGCTGTCGG \\
&
\end{tabular}

Tris-buffered saline (TBS)+tween-20 (TBST, Tween-20: TBS $=1: 1,000)$ for $5 \mathrm{~min}$ and blocked using $5 \%$ skimmed milk powder at $4{ }^{\circ} \mathrm{C}$ overnight. The samples were then incubated for $1 \mathrm{~h}$ with the following primary antibodies, rabbit anti-mouse polyclonal antibodies to JAK2 p-Y1007 + Y1008 (1:2,000; ab32101), STAT3 p-Y705 (1:1,000; ab76315), sphingosine-1-phosphate receptor 1 (S1PR1; 1:5,000; ab11424), NCALD (1:2,000; PA5-76322), C/ EBP $\beta$ (1:2,000; ab32358) and GAPDH (1:5,000; ab8245). This was followed by three TBST washes ( $5 \mathrm{~min} / \mathrm{wash}$ ). The samples were then incubated with oscillation at room temperature for $40 \mathrm{~min}$ in the secondary antibody, horseradish peroxidase (HRP)-labeled goat anti-rabbit IgG (1:20,000, BA1054, BOSTER Inc.). The PVDF membrane was then washed three times with $25 \mathrm{~mL}$ TBST (5 min each time) and incubated with electrogenerated chemiluminescence (ECL) solution (ECL808-25, Biomiga, San Diego, CA, USA) for $1 \mathrm{~min}$. Excess liquid was removed, and the PVDF membrane was exposed to X-ray film. The net density value of the bands was calculated by ImagePro Plus 6.0, with GAPDH as an internal reference band. Western blot analysis was also performed on the rBMECs and rAstrocytes. All the experiments were repeated three times.

\section{QRT-PCR}

The expression levels of miR-181b in rats in the sham, sepsis, Stattic, and sepsis + anta-miR-181b groups, as well as in rBMECs and rAstrocytes treated with sham and sepsis rat serum were analyzed. The expression of S1PR1 and $N C A L D$ was also assessed. Total RNA was extracted from tissues and cells using TRIzol reagent. The primers of miR181b, S1PR1, NCALD, U6, and GAPDH were designed and synthesized by Sangon, Shanghai, China (Table 1).

Amplification of miR-181b was performed using PrimeScript ${ }^{\mathrm{TM}}$ RT reagent Kit (Takara) according to the manufacturer's instructions in a Stratagene Mx3000P machine (MX3000P, Stratagene, USA). The relative amount of each miRNA and messenger RNA (mRNA) to the reference gene (U6 RNA) was calculated using the 2-(ctmiRNA-ctU6) equation. As for mRNAs, a total of 1 $\mu \mathrm{g}$ RNA was employed as the template for transcription, which was conducted using the Bestar qPCR RT Kit (DBI Bioscience) in a $20 \mu \mathrm{L}$ system. Reactions were held at 94 ${ }^{\circ} \mathrm{C}$ for $2 \mathrm{~min}$, followed by 40 cycles of amplification at 94 ${ }^{\circ} \mathrm{C}$ for $20 \mathrm{~s}, 58^{\circ} \mathrm{C}$ for $20 \mathrm{~s}$, and $72{ }^{\circ} \mathrm{C}$ for $20 \mathrm{~s}$ in a Stratagene Mx3000P machine (MX3000P, Stratagene, USA). The relative amount of each miRNA and mRNA to the reference gene $(\mathrm{GAPDH})$ was measured using the $2^{-\Delta \Delta \mathrm{Ct}}$ method. All experiments were repeated three times.

\section{Chromatin immunoprecipitation-quantitative PCR (ChIP- QPCR)}

The ChIP-QPCR technique is used to study whether a transcription factor binds with the promoter of a certain gene, including miRNAs. In the present study, the binding 
and enrichment of JAK2/STAT3 with the miR-181b promoter region was studied in $\mathrm{rBMEC}$ treated with $10 \%$ serum from sham and septic rats for $24 \mathrm{~h}$. After stimulation with the serum, the cells were treated with ChIP-QPCR assay, as detailed in Supplementary material 1 (Appendix 1).

\section{Dual-luciferase reporter gene assay}

The target genes of miR-181b, including S1PR1 and NCALD, were predicted with TargetScan (http://www. targetscan.org/vert_72/). A dual-luciferase reporter gene assay was used to verify whether S1PR1 and NCALD were the direct target genes of miR-181b. p-S1PR1-wild-type (WT) and p-NCALD-WT, as well as the full length $3 '-$ UTRs of S1PR1 and NCALD, were cloned and amplified using psiCHECK-2 (Promega, Madson). The pS1PR1mutant (MUT) and pNCALD-MUT were constructed by rite-directed mutagenesis. The miR-181b mimic and negative control (miR-181b NC) were co-transfected into HEK-293 T cells (CRL-1415, American Type Culture Collection, Manassas, VA) with luciferase reporter vector. A fluorescence detector (Glomax20/20, Promega, Collin, 1989) was employed to observe fluorescence intensity.

\section{Cell adbesion assay}

Cell adhesion of rBMECs treated with $10 \%$ serum from sham, sepsis, and sepsis + anta-miR-181b rats was evaluated according to the protocol of the BestBio Inc. Cell Adhesion Assay (cat. BB-48120, Shanghai, China). After the assessment, the cell adhesion rate was calculated as (rBMECs OD from sepsis-blank cell OD)/ (rBMECs OD from shamblank cell OD) $\times 100$.

\section{Statistical analysis}

GraphPad Prism 8 (GraphPad Software, San Diego, CA, USA) was used for statistical analysis and visualization. Data were expressed as mean \pm standard deviation. Comparisons between two groups were conducted using the $t$-test, and results were considered statistically significant when $\mathrm{P}<0.05$.

\section{Results}

miR-181b and $\mathcal{F A K}$-STAT3-related proteins were highly expressed in septic rats

In the present study, 10 rats were studied in each group.
The experiment revealed dramatically increased levels of serum D-lactate, TNF- $\alpha$, IL-6, and IL- 8 in the septic rats compared with the sham rats $(\mathrm{P}<0.001$; Figure $1 A)$. Moreover, the levels of the pro-inflammatory cytokines as TNF- $\alpha$, IL-6, and IL- 8 were significantly higher in the brains of the septic rats compared to those of the sham rats $(\mathrm{P}<0.01$; Figure $1 B)$. Extravasation of $\mathrm{EBD}$ was used to assess changes in the vascular permeability of the brain. Figure $1 C$ showed that EBD was significantly increased in the septic rats compared to the sham rats, indicating severe $\mathrm{BBB}$ damage in the septic rats. Taken together, these results demonstrated the successful construction of a rat sepsis model, which was the basis of our current study.

Using western blot analysis, we assessed the expression of $\mathrm{C} / \mathrm{EBP} \beta$ and other key proteins in the JAK/STAT3 signaling pathway, including JAK2, p-JAK2, STAT3, and p-STAT3. The results demonstrated that expression of p-JAK2, p-STAT3, and C/EBP $\beta$ was significantly elevated in the septic rats compared to the sham rats $(\mathrm{P}<0.001$; Figure 1D, E). The expression of miR-181b in the cerebral cortex and serum of the sepsis and sham rats was also assessed. The expression level of miR-181b in the septic rats was significantly higher than that in the sham rats $(\mathrm{P}<0.001$; Figure $1 F)$.

\section{STAT3 inhibitor relieved BBB damage and downregulated miR-181b in vitro}

To investigate the effect of miR-181b regulation by STAT3 on the BBB, rBMECs and rAstrocytes were incubated with $10 \%$ serum from sham or septic rats for $24 \mathrm{~h}$. Hoechst staining demonstrated that apoptosis was enhanced in rBMECs and rAstrocytes treated with serum from septic rats compared to those treated with serum from sham rats (Figure 2A).

As shown in Figure $2 B, C$, cell apoptosis in rBMECs and rAstrocytes exposed to serum from septic rats was more severe than that in cells exposed to sham serum, and the expression levels of p-JAK2 and p-STAT3 were also higher in the septic rats. The protein expressions of JAK2, p-JAK2, STAT3, p-STAT3, and C/EBP $\beta$ in rBMECs and rAstrocytes from sham and septic rats were also assessed through western blot analysis. The result showed that serum from the septic rats significantly stimulated p-JAK2, p-STAT3, and C/EBP $\beta$ expression in both $\mathrm{rBMECs}$ and rAstrocytes compared with sham serum $(\mathrm{P}<0.001$; Figure $2 D, E)$. The expression of miR-181b was also significantly higher in rBMECs and rAstrocytes cells treated with 
A

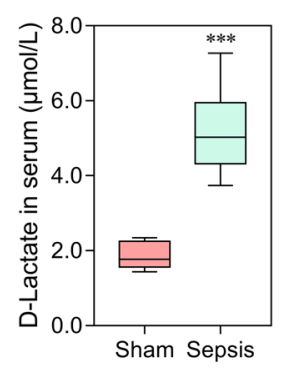

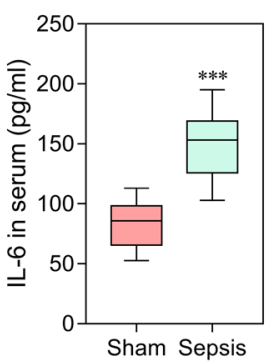
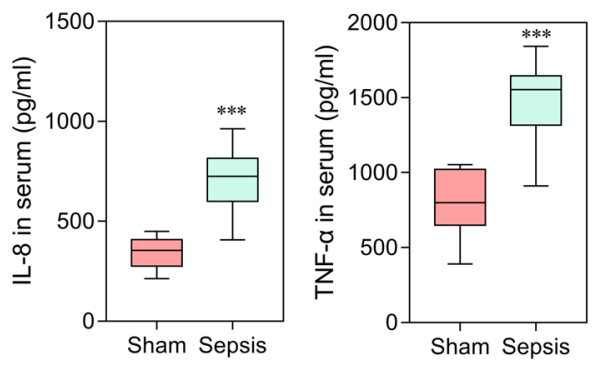

B

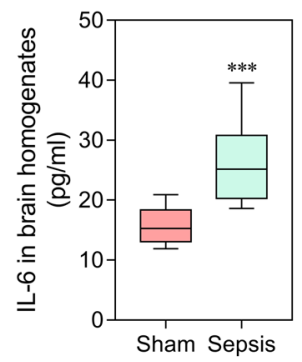

D

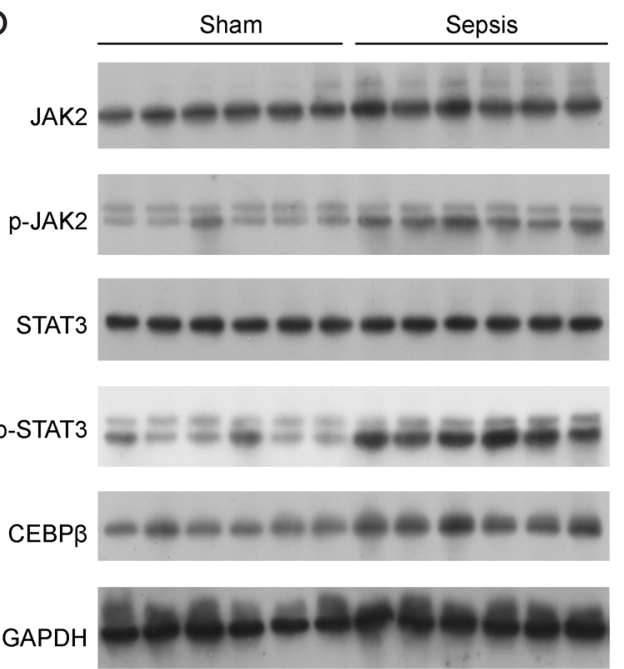

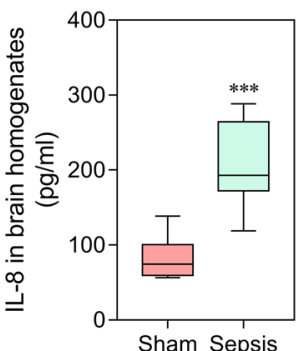

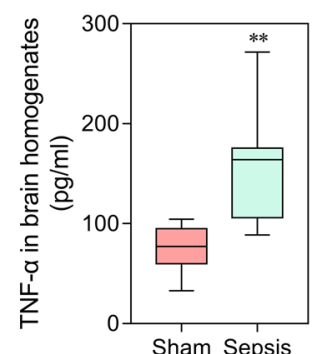

$\mathrm{E}$
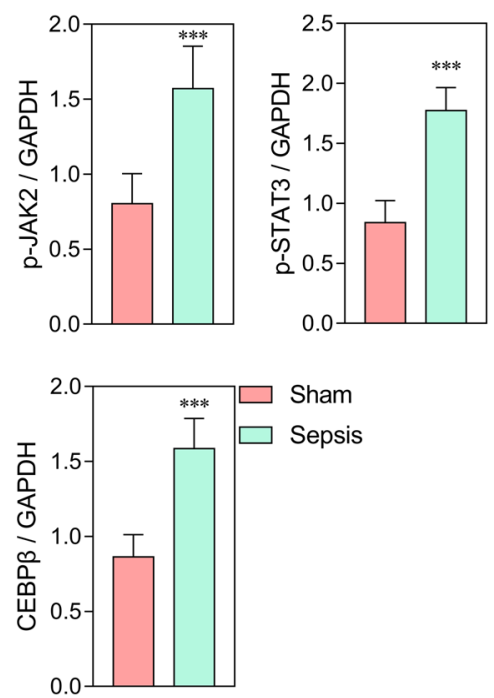

C
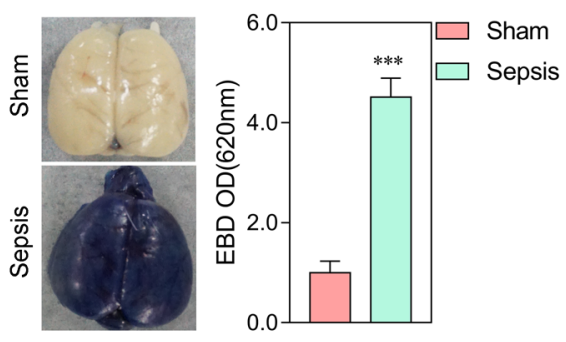

$\mathrm{F}$
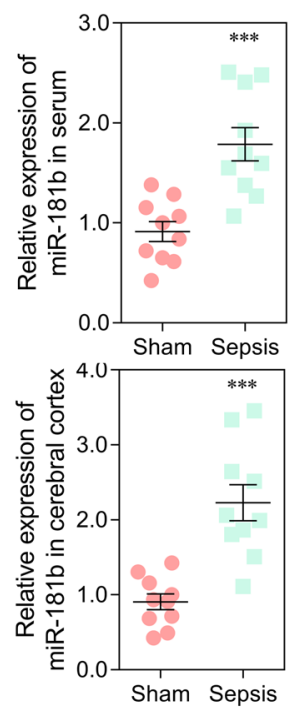

Figure 1 Rat model construction and expression of miR-181b and JAK-STAT3-related proteins. (A,B) Serum D-lactate, TNF- $\alpha$, IL-6, and IL-8 in serum and brain homogenates of septic and sham rats were assessed by ELISA; (C) Brain vascular permeability was detected by EBD leakage in septic and sham rats $(n=3)$. Extracted dye contents in the formamide extracts were quantified at $620 \mathrm{~nm}$; $(\mathrm{D}, \mathrm{E})$ The protein expression levels of C/EBP $\beta$, JAK2, p-JAK2, STAT3, and p-STAT3 were analyzed; (F) The relative expression levels of miR-181b in the serum and cerebral cortex of sham and septic rats were analyzed. **, $\mathrm{P}<0.01 ;{ }^{* * *}, \mathrm{P}<0.001$. C/EBP $\beta$, transcription factor CCAAT enhancer binding protein $\beta$; EBD, Evans blue dye; ELISA, enzyme-linked immunosorbent assay. GAPDH, glyceraldehyde-3-phosphate dehydrogenase; IL-6, interleukins 6; IL-8, interleukins 8; JAK2, Janus kinase 2; miR-181b, microRNA-181b; p-JAK2, phosphorylation JAK2; p-STAT3, phosphorylation STAT3; STAT3, signal transducer and activator of transcription 3; TNF- $\alpha$, tumor necrosis factor alpha. 

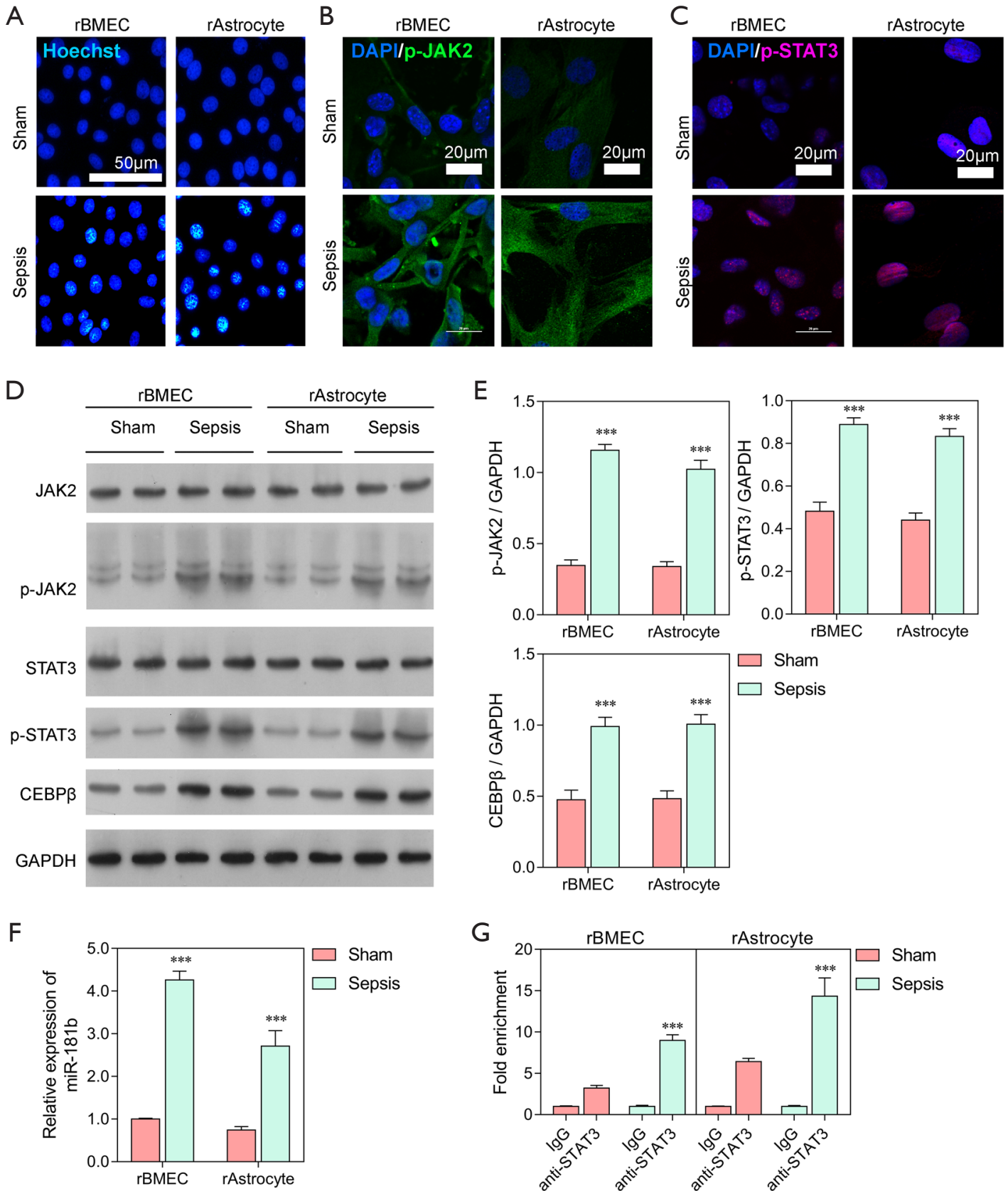

Figure 2 The effects of STAT3 on miR-181b in rBMECs and rAstrocytes treated with serum from sham and septic rats. (A) Hoechst 33258 staining demonstrating apoptosis of rBMECs and rAstrocytes after treatment with serum from sham or septic rats. The nucleus of normal cells were dyed dark and apoptosis cells were dyed light; (B,C) Immunofluorescence and TUNEL staining for p-JAK2 and p-STAT3 in rBMECs and rAstrocytes treated with sham and sepsis rat serum (blue, DAPI; green, p-JAK2; red, p-STAT3); (D,E) Western blot analysis of JAK2 in rBMECs and rAstrocytes treated with serum from sham and septic rats; (F) Expression of miR-181b in rBMECs and rAstrocytes treated with serum from sham and septic rats; $(\mathrm{G})$ ChIP-QPCR analysis of miR-181b binding with STAT3. C/EBP $\beta$, transcription factor CCAAT enhancer binding protein $\beta .{ }^{* *}, \mathrm{P}<0.001$. ChIP-QPCR, chromatin immunoprecipitation-quantitative PCR; DAPI, 4',6-diamidino-2-phenylindole; GAPDH, glyceraldehyde-3-phosphate dehydrogenase; IgG, immunoglobulin G; JAK2, Janus kinase 2; miR-181b, microRNA-181b; p-JAK2, phosphorylation JAK2; p-STAT3, phosphorylation STAT3; STAT3, signal transducer and activator of transcription 3; TUNEL, terminal transferase mediated UTP nick end-labeling kit. 
A
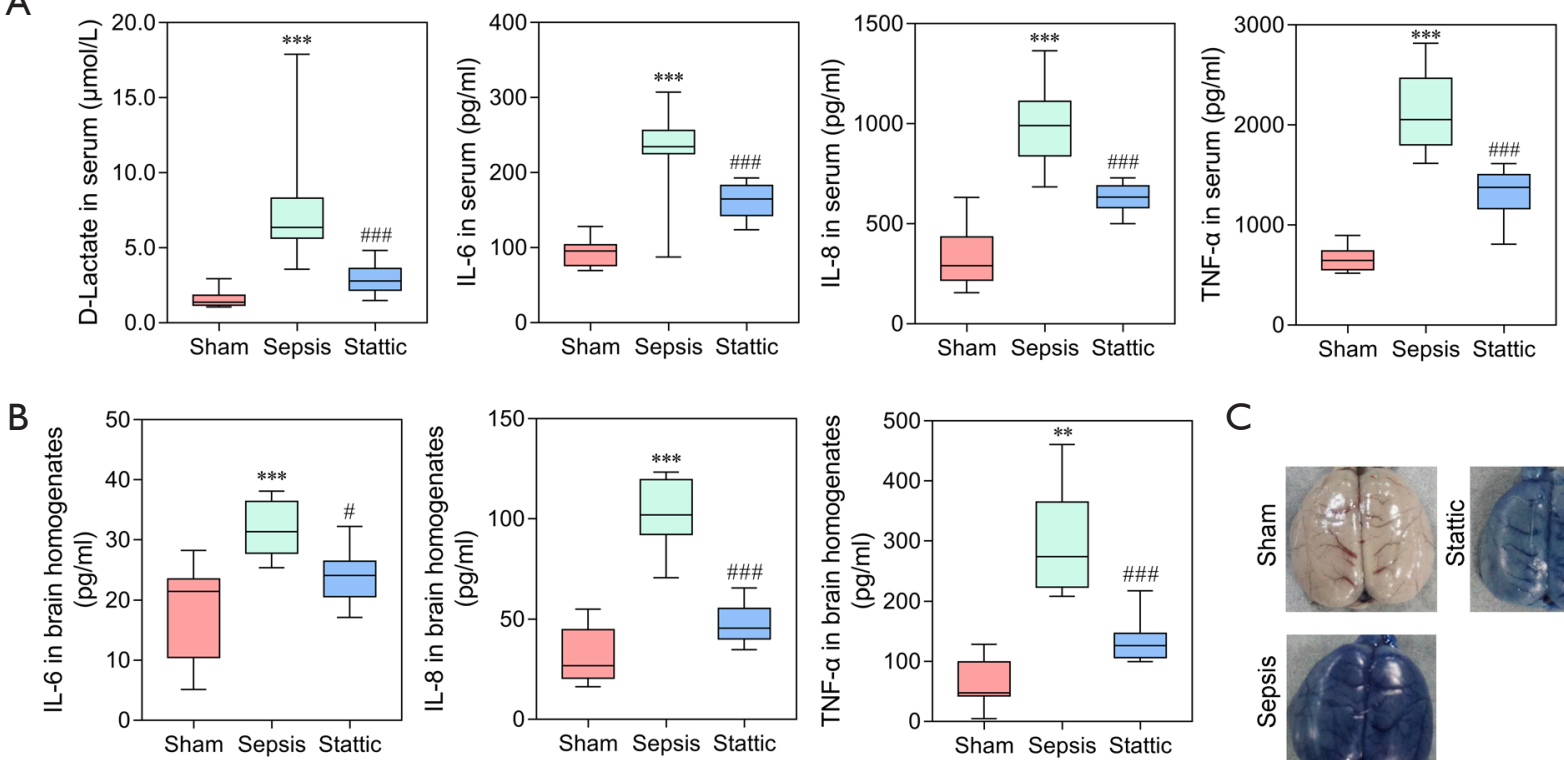

C
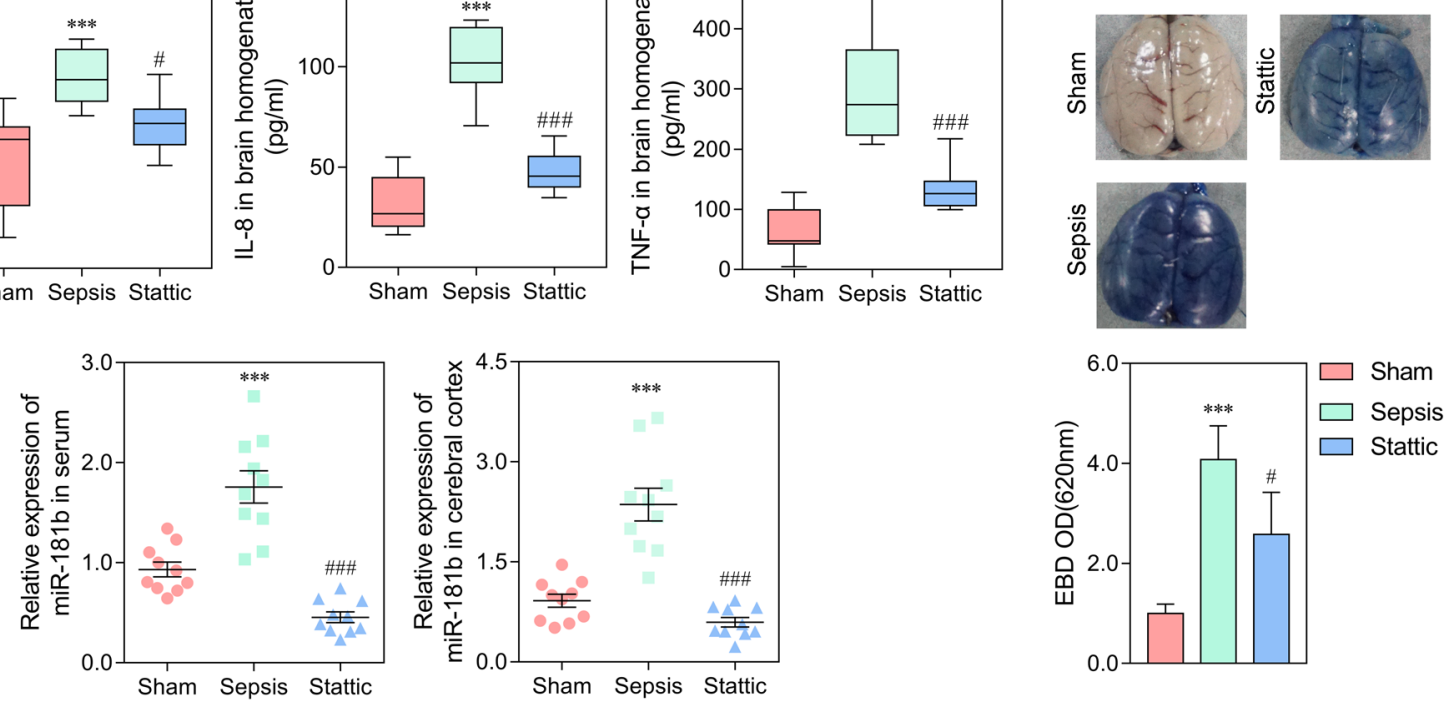

Figure 3 STAT3 inhibitor reduced damage in the BBB through downregulating miR-181b in vivo. (A,B) Assessment of serum D-lactate, TNF- $\alpha$, IL-6, and IL-8 in the serum and brain homogenates of sham, septic, and Stattic rats by ELISA; (C) Brain vascular permeability as detected by EBD leakage in the sepsis, Stattic, and sham rats $(n=3)$. Extracted dye contents in the formamide extracts were measured at $620 \mathrm{~nm}$; (D) The relative expression of miR-181b in the serum and cerebral cortex of sham, septic, and Stattic rats. ${ }^{* *}, \mathrm{P}<0.01 ;{ }^{* * *}, \mathrm{P}<0.001$

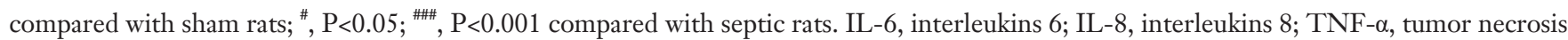
factor alpha; EBD, Evans blue dye; miR-181b, microRNA-181b.

sepsis rat serum than in those treated with serum from the sham rats $(\mathrm{P}<0.001$; Figure $2 F)$. ChIP-QPCR analysis was performed to detect the binding and enrichment of the JAK2/STAT3 signal core transcription complex in the miR-181b promoter region. As shown in Figure 2G, the fold enrichment of STAT3 was significantly higher in cells treated with serum from the sepsis rats, in which the expression of miR-181b was also significantly elevated. Taken together, these results indicated that STAT3 was involved in the BBB and significantly upregulated the expression of miR-181b.

\section{STAT3 inbibitor relieved BBB damage and downregulated miR-181b in vivo}

To further confirm the relationship between STAT3 and miR-181b, an in vivo experiment involving sham, sepsis, and Stattic (sepsis + STAT3 inhibitor, $20 \mathrm{mg} / \mathrm{kg}$ ) rats was conducted. The serum levels of D-lactate, IL-6, IL-8, and TNF- $\alpha$ in the septic rats were significantly higher than those observed in the sham and Stattic rats $(\mathrm{P}<0.001$; Figure $3 A)$. Similar results were observed in brain homogenates $(\mathrm{P}<0.001$; Figure $3 B)$. As shown in Figure $3 C$, EBD was significantly increased in the brains of septic rats compared 
A

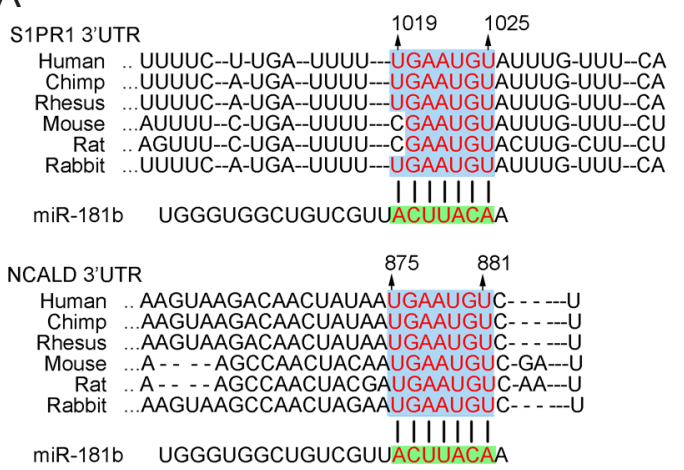

B

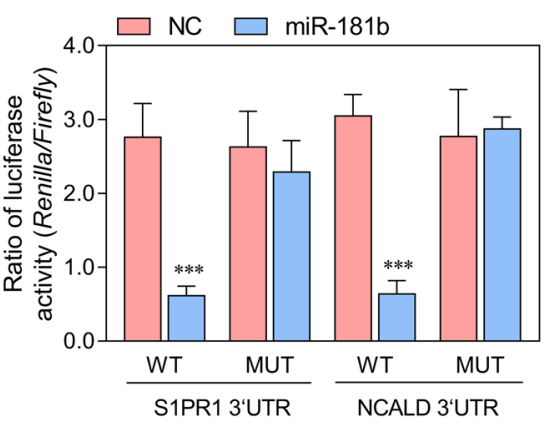

C

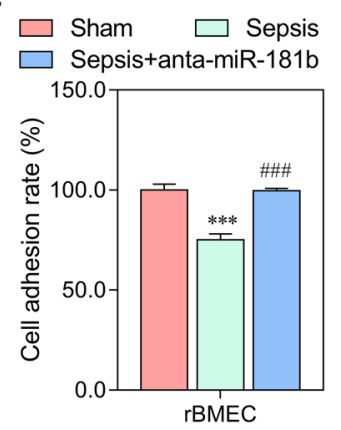

Figure 4 S1PR1 and NCALD were negatively regulated by miR-181b. (A) The predicted relationships of the genes S1PR1 and NCALD with miR-181b, as shown by bioinformatics prediction website. (B) The targeted relationships of S1PR1 and NCALD with miR-181b. (C)

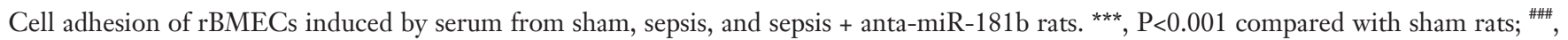
$\mathrm{P}<0.01$ compared with septic rats. miR-181b, microRNA-181b. MUT, mutant. NC, negative control. WT, wild-type. S1PR1, sphingosine1-phosphate receptor 1. NCALD, neurocalcin delta.

to those of both the sham $(\mathrm{P}<0.001)$ and Stattic $(\mathrm{P}<0.05)$ rats, demonstrating that Stattic could reduce the damage to the BBB. Moreover, the expression of miR-181b in the serum and cerebral cortex was shown to be significantly downregulated in the Stattic and sham rats compared to that in the septic rats (Figure $3 D, \mathrm{P}<0.001$ ). Taken together, we concluded that the STAT3 protected against BBB damage by downregulating the expression of miR-181b.

\section{S1PR1 and NCALD were negatively regulated by miR- $181 b$}

Using miRNA prediction software and a dual-luciferase reporter gene assay, a detailed binding region between miR-181b and gene sequences from S1PR1 and NCALD was found, which indicated that S1PR1 and NCALD might indeed be the target genes of miR-181b (Figure 4A). As shown in Figure $4 B$, compared with the negative control group, the luciferase activity of S1PR1-WT and NCALDWT was significantly inhibited by miR-181b $(\mathrm{P}<0.05)$, whereas the same activity was not inhibited in S1PR1MUT and NCALD-MUT. These results suggest that miR$181 \mathrm{~b}$ might specifically bind to the 3'-UTRs of S1PR1 and $N C A L D$, and simultaneously downregulate the expression of S1PR1 and NCALD at the post-transcriptional level. In comparison with cells treated with sham serum and sepsis + anta-miR-181b serum, cell adhesion was significantly decreased in cells treated with serum from the septic rats (Figure 4C), confirming the inhibitory effect of miR-181b on S1PR1.

\section{miR-181b promoted impairment of the BBB through the downregulation of S1PR1 and NCALD in vitro}

To further confirm the regulation of miR-181b on BBB damage, the in vitro experiments using rBMECs and rAstrocytes were conducted. Cells were treated with $10 \%$ serum from sham, sepsis, and sepsis + anta-miR-181b rats. After treatment for $24 \mathrm{~h}$, cell apoptosis was assessed by Hoechst staining. As shown in Figure 5A, cell apoptosis was promoted by serum from the septic rats and inhibited by serum from the sepsis + anta-miR-181b and sham rats, especially in rBMECs. As mentioned above, it is likely that miR-181b negatively regulates the gene and protein expression of both S1PR1 and NCALD, which were significantly downregulated in the present study $(\mathrm{P}<0.001$; Figure $5 B, C)$. anta-miR-181b inhibitor significantly increased the gene and protein expression levels of S1PR1 and NCALD in both rBMECs and rAstrocytes in serum from the septic rats $(\mathrm{P}<0.001$; Figure $5 B, C)$. No significant differences were found in gene or protein expression between cells treated with serum from the sham and sepsis + anta-miR-181b rats $(\mathrm{P}>0.05$; Figure $5 B, C)$. Immunofluorescence and TUNEL staining for NCALD in rBMECs and rAstrocytes treated with serum from the sham, sepsis, and sepsis + anta-miR-181b rats revealed that sepsis rat serum promoted cell apoptosis and downregulated the expression of NCALD, while sepsis + anta-miR-181b 

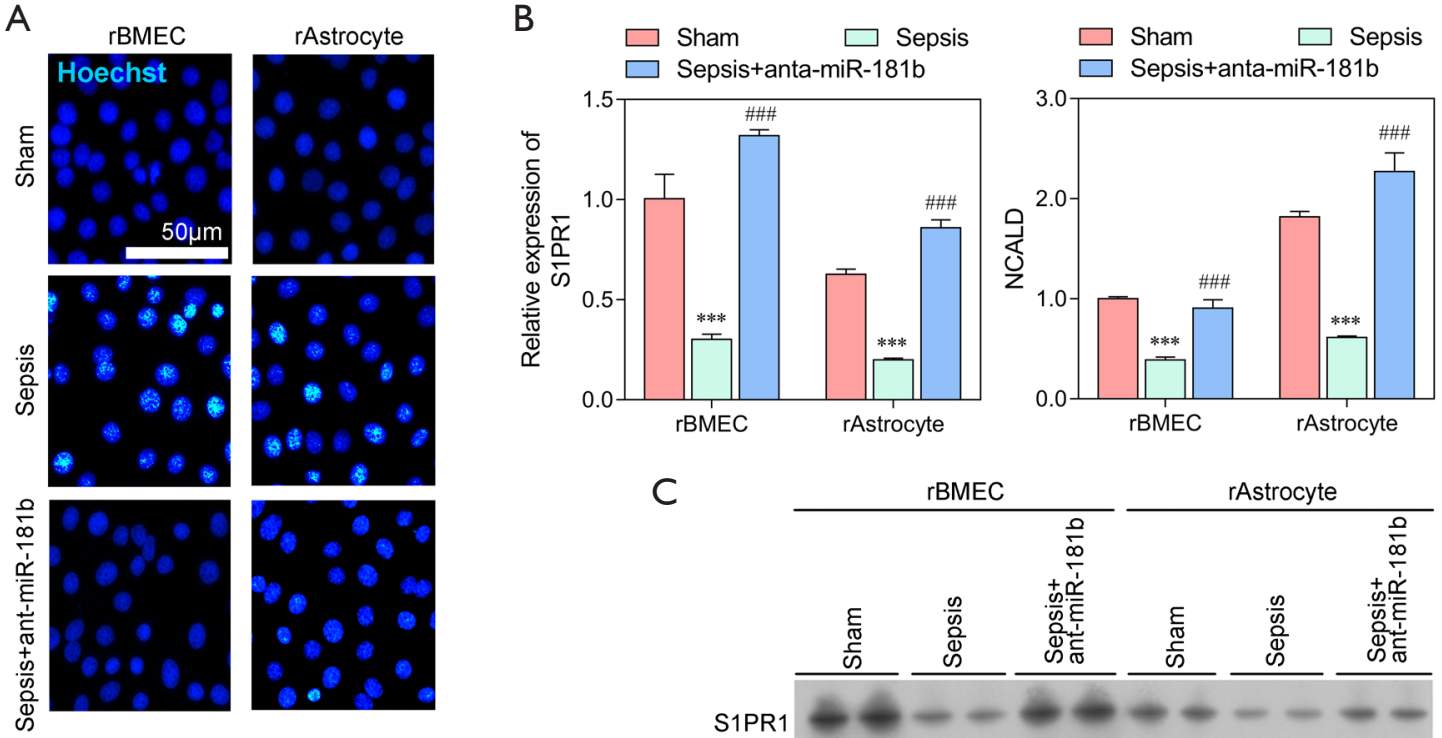

C
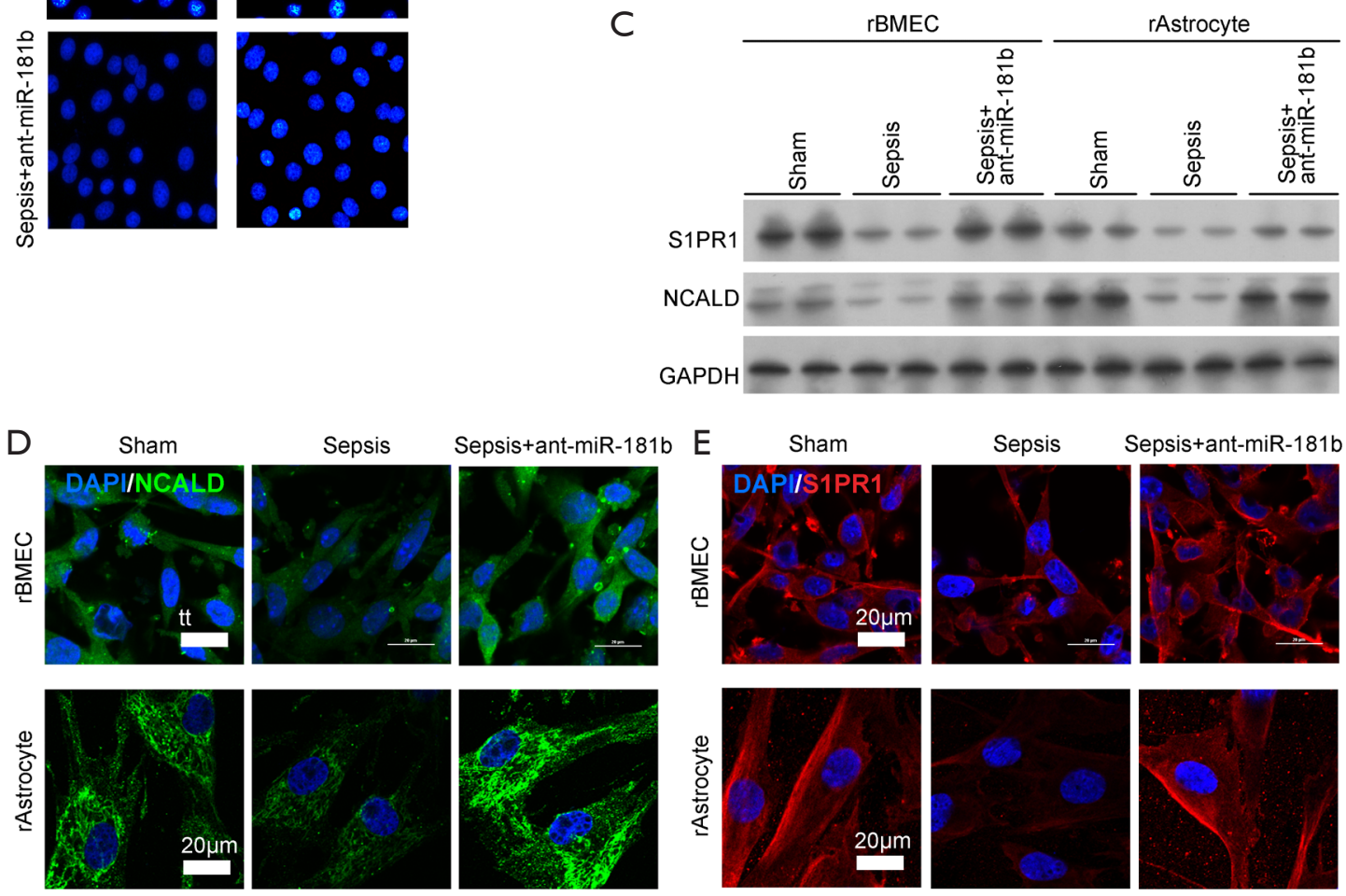

Figure 5 miR-181b promotes BBB impairment through downregulating S1PR1 and NCALD in vitro. (A) The effects of sham, sepsis, and sepsis + anta-miR-181b rat serum on apoptosis in rBMECs and rAstrocytes as shown by Hoechst 33258 staining. The nucleus of normal cells were dyed dark and the apoptosis cells were dyed light; (B,C) The gene and protein expression of S1PR1 and NCALD in rBMECs and rAstrocytes treated with serum from sham, septic, and sepsis + anta-miR-181b rats; (D,E) Immunofluorescence staining for S1PR1 and NCALD in rBMECs and rAstrocytes treated with serum from sham, sepsis, and sepsis + anta-miR-181b rats (blue, DAPI; green, S1PR1; red, NCALD). ***, $\mathrm{P}<0.001$ compared with sham rats; ${ }^{\# \#}, \mathrm{P}<0.01$ compared with septic rats. BBB, blood-brain barrier. S1PR1, sphingosine1-phosphate receptor 1. NCALD, neurocalcin delta. DAPI, 4',6-diamidino-2-phenylindole. miR-181b, microRNA-181b. GAPDH, glyceraldehyde-3-phosphate dehydrogenase. TUNEL, terminal transferase-mediated UTP nick end-labeling.

increased cell proliferation and NCALD expression (Figure 5D). Similarly, the expression of S1PR1 was significantly downregulated in rBMECs and rAstrocytes treated with serum from the septic rats compared with sham rat serum. Sepsis + anta-miR-181b serum increased S1PR1 expression back to levels observed in cells treated with sham serum
(Figure 5E).

miR-181b promoted BBB impairment by downregulating S1PR1 and NCALD in vivo

Sham, sepsis, and sepsis + anta-miR-181b rat models were 


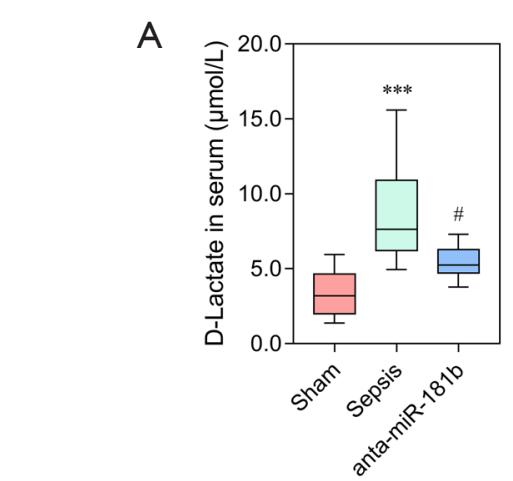

B

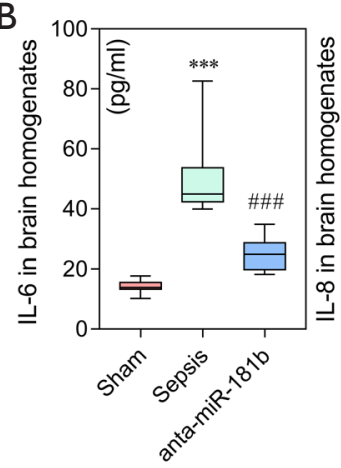

$\mathrm{D}$

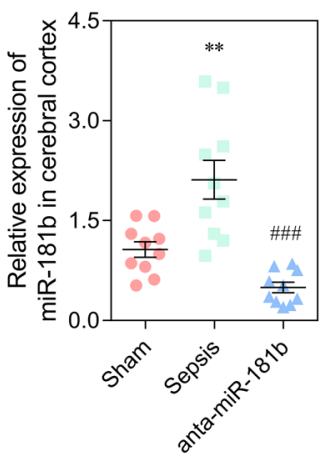

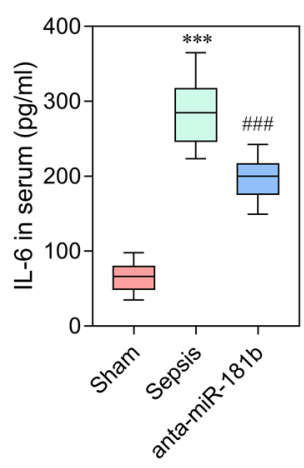
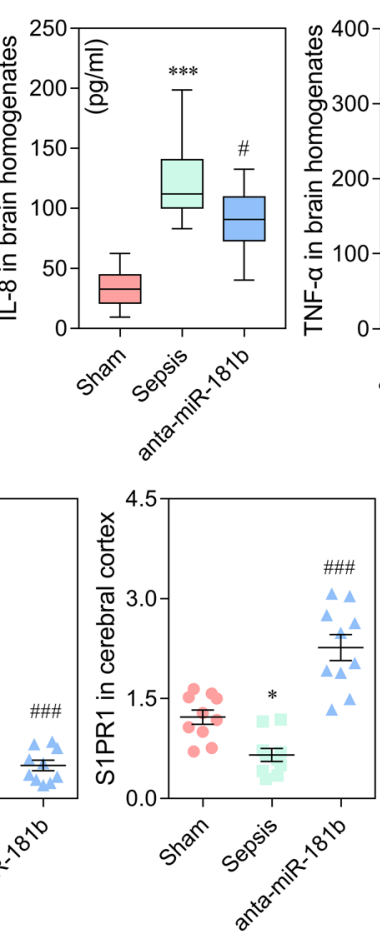

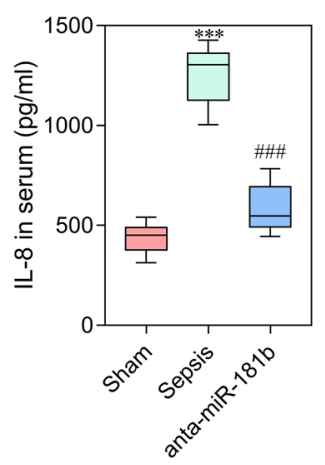

C
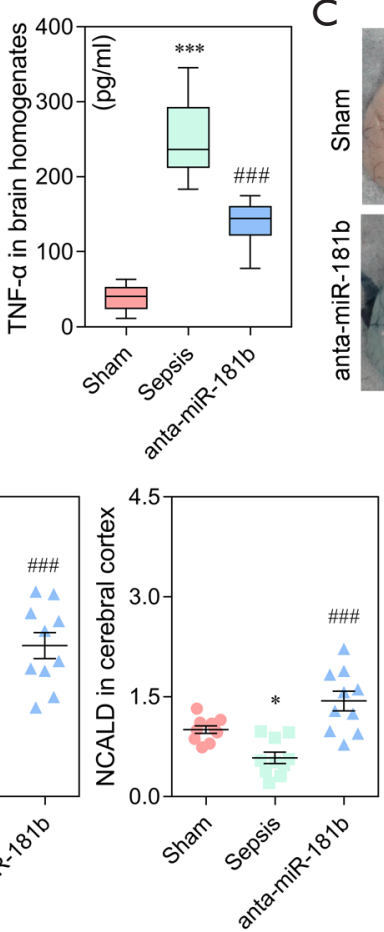

$E$
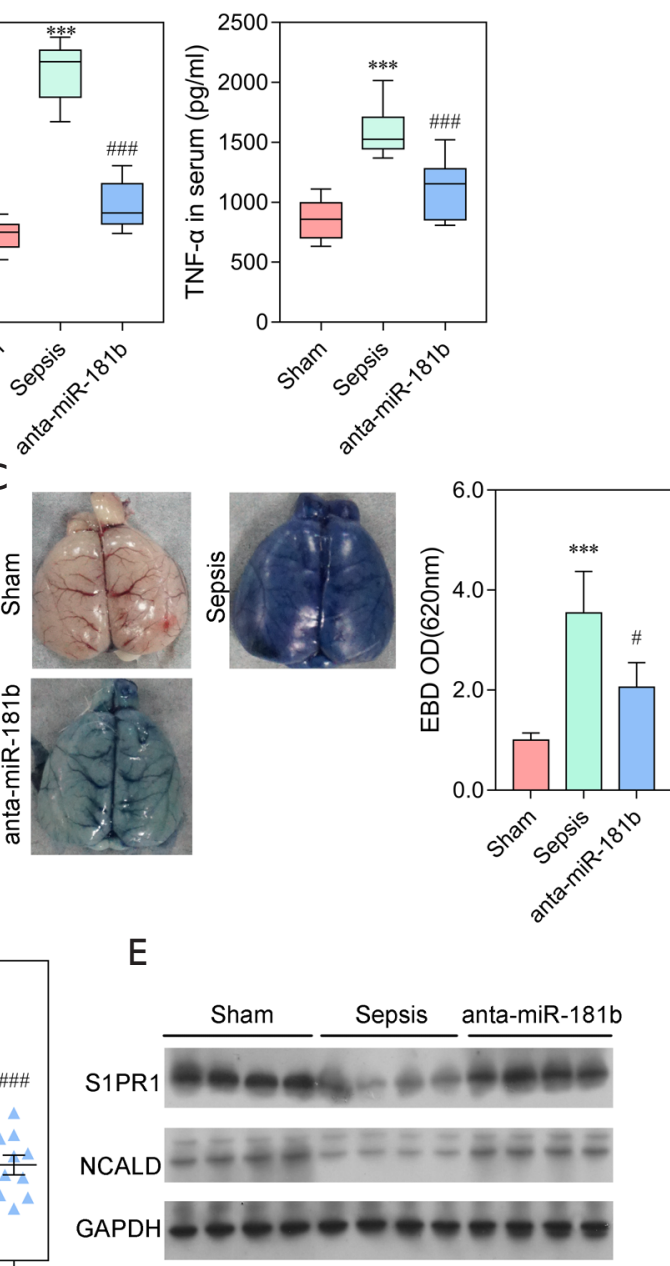

Figure 6 miR-181b promoted BBB impairment by downregulating S1PR1 and NCALD . (A,B) Serum D-lactate, TNF- $\alpha$, IL-6, and IL-8 in serum and brain homogenates of sham, sepsis, and sepsis + anta-miR-181b rats were assessed by ELISA; (C) Brain vascular permeability was detected with EBD leakage in the sham, sepsis, and sepsis + anta-miR-181b rats (n=3); (D) Gene expression levels of miR-181b, S1PR1 and NCALD in the cerebral cortex; (E) The protein expression levels of S1PR1 and NCALD. *, $\mathrm{P}<0.05 ;{ }^{* *}, \mathrm{P}<0.01 ;$ ***, $\mathrm{P}<0.001$ compared with Sham; ${ }^{\#}, \mathrm{P}<0.05$ compared with Sepsis; ${ }^{\# \#}, \mathrm{P}<0.001$ compared with Sepsis. BBB, blood-brain barrier; IL-6, interleukins 6; IL-8, interleukins 8; TNF- $\alpha$, tumor necrosis factor alpha; EBD, Evans blue dye; S1PR1, sphingosine-1-phosphate receptor 1; NCALD, neurocalcin delta.

constructed. The serum levels of D-lactate, IL-6, IL-8, and TNF- $\alpha$ in the septic rats were significantly higher than those observed in the sham and sepsis + anta-miR-181b rats $(\mathrm{P}<0.001$; Figure $6 A)$. Similar results were observed in rat brain tissue $(\mathrm{P}<0.001 ;$ Figure $6 B)$. In the septic rats, the EBD content was significantly decreased after injection with anta-miR-181b inhibitor $(\mathrm{P}<0.05$; Figure $6 C)$. The gene and protein expression of S1PR1 and NCALD in the sepsis + anta-miR-181b rats was similar to that observed in the sham rats, both of which were significantly higher than that observed in the septic rats $(\mathrm{P}<0.001$; Figure $6 D, E)$. As expected, the expression of miR-181b was significantly increased in the septic rats, but downregulated in rats injected with anta-miR-181b (Figure 6D). Taken together, 
these results demonstrated that the miR-181b inhibitor increased the expression of S1PR1 and NCALD, thereby inhibiting damage to the BBB. This strongly suggests that negative regulation of S1PR1 and NCALD by miR-181b is a mechanism of BBB damage.

\section{Discussions}

Sepsis, which is often caused by infections, is characterized by severe systemic inflammation responses and has a high rate of morbidity. Unfortunately, there is also a high incidence of severe SAE (26). As SAE is caused by damage to the $\mathrm{BBB}$, protecting the $\mathrm{BBB}$ has become a vital objective in the treatment of sepsis patients. However, the molecular mechanisms of BBB impairment in sepsis patients with SAE are poorly understood. In-depth study of miRNAs has revealed the utility of serum miRNAs as biomarkers for a range of diseases, including Alzheimer's disease (27), Parkinson's disease (28), and amyotrophic lateral sclerosis (29). Studies in rats have demonstrated that miR-181b promotes immunosuppression during sepsis through its inhibitory effect on bone marrow-derived suppressor cells, ultimately resulting in death $(22,23)$. Cause inflammatory cytokines can be released by JAK/STAT signaling pathway, we performed this study to investigate whether miR-181b promotes damage to the BBB during sepsis via the JAK/ STAT signaling pathway. Notably, this was the first report on JAK/STAT signaling pathway regulating BBB by miR$181 \mathrm{~b}$ in septic rat.

In the present study, D-lactate, TNF- $\alpha$, IL-6, and IL-8 were selected as the pro-inflammatory cytokines that characterize sepsis (30-33). These cytokines in the central nervous system can lead to cerebral dysfunction in sepsis survivors. A significant increase in TNF- $\alpha$, IL- 6 , and IL-8 was found in the serum and brains of septic rats compared with those of the sham rats. Monitoring blood D-lactate levels can reflect intestinal permeability changes and the extent of damage (34). Studies have also revealed a higher content of TNF- $\alpha$, IL- 6 , and IL- 8 in sepsis serum than in serum from sham-operated rats $(14,35)$, which was consistent with the findings of the present study. In addition to serum, the levels of TNF- $\alpha$, IL- 6 , and IL- 8 have also been reported to be increased in the intestines (36). In summary, the experiments measuring serum and brain levels of TNF- $\alpha$, IL-6 and IL-8, as well as the results of the EBD, reflected the successful establishment of a sepsis group, and formed the basis for our further experiments.

In the present study, the JAK/STAT signaling pathway was activated, just as reported by Chang et al. (12). Moreover, the expression of miR-181b was also upregulated, and was regulated by JAK/STAT signaling pathway. Halytskiy (37) also demonstrated that STAT3 activity facilitates hyperexpression of miRNAs miR-155, miR-181 and miR-21, which was agreed with our present study. With the addition of STAT3 inhibitor and antamiR-181b, the miR-181b was down-regulated and the permeability of $\mathrm{BBB}$ is also improved. Enough evidence indicated that miR-181b inhibitor can promote the permeability of $\mathrm{BBB}$, which might be an ideal therapy for SAE.

Other than miR-181b, some other factors can also affect $\mathrm{BBB}$. The protein kinase $\mathrm{C}$-delta inhibition protects blood-brain barrier from sepsis-induced vascular damage by preventing events that lead to vascular damage and inflammation-induced tissue damage (38). Margotti et al. (39) found that aging potentiated BBB permeability in sepsis, which possibly triggered an increase in neutrophil infiltration and, consequently, an increase in oxidative stress. Tetramethylpyrazine alleviated the degree of bloodbrain barrier destruction by increasing the expression of tight junction-related proteins claudin-5 and occluding in the brain tissue and increased the expression of the ZO-1, Occludin, and Claudin-5 genes (40).

JAK/STAT signaling pathway has been shown related to the release of various cytokines and inflammatory mediators and involved in the regulation of immune response in sepsis $(7,8)$. Some other miRNAs, as miRNA-30a and miR-155 were also reported regulated sepsis-induced liver injury through regulating JAK/STAT signaling pathway $(14,15)$. In the present study, we confirmed the relationship between miR-181b, JAK/STAT signaling pathway on influencing permeability of BBB. Moreover, the anta-miR-181b might be an ideal therapy for SAE.

Thereafter, we focused on the target genes of miR$181 \mathrm{~b}$ that might play essential roles in influencing BBB impairment, and two genes of S1PR1 and NCALD were identified. Sphingosine-1-phosphate (S1P) and S1PR1 are active regulators of sepsis-relevant signaling events and are potential therapeutic targets and biomarkers for sepsis (41). As reported by Gaengel and colleagues (42), activated S1PR1 signal inhibits angiogenic sprouting and enhances cell-to-cell adhesion. The results in our present study showed miR-181b suppressed S1PR1 expression and inhibited cell adhesion in rBMECs. We therefore suggest that increased expression of miR-181b downregulates S1PR1, leading to decreased cell adhesion and damage to 
the BBB.

Another target gene of miR-181b, NCALD, was also downregulated by miR-181b. NCALD, HPCA (Hippocalcin), and S100B are neuronal calcium sensor proteins that confer calcium sensitivity to neuronal and non-neuronal cells (43). S100B can maintain the BBB and regulate circadian rhythm or sleep disorders. However, to date, NCALD has only been shown to regulate circadian rhythm. In the present study, NCALD was also targeted and downregulated by miR-181b in septic rats. Therefore, we suspected that miR-181b prevented the neurons from entering rBMECs and rAstrocytes by downregulating NCALD, thus causing severe BBB impairment in sepsis. As reported by Dalvi and colleagues (44), miRNAs are packaged into exosomes and carried to surrounding cells. Thus, it is possible that miR-181b may enter cells by way of exosomes. However, the mechanism of S1PR1 and NCALD need further study.

\section{Conclusions}

In conclusion, the present study demonstrated that JAK2/ STAT3 signaling pathway induced expression of miR-181b, which promoted BBB impairment in rats with sepsis by downregulating S1PR1 and decreasing BBB cell adhesion., suggesting JAK2/STAT3/miR-181b axis as therapeutic target in protecting against sepsis-induced BBB damage. Moreover, S1PR1 also plays a role in BBB damage by reducing cell adhesion. Our present study is the first to describe the JAK/STAT3-miR-181b-S1PR1 mechanism in BBB damage in sepsis.

\section{Acknowledgments}

Funding: This study was supported by the National Natural Science Foundation of China (No. 81701875), Science and Technology Program of Guangzhou (No. 201804010379 and No. 201904010039) and Medical Scientific Research Foundation of Guangdong Province (No. A2017284 and No. A2020540).

\section{Footnote}

Reporting Checklist: The authors have completed the ARRIVE reporting checklist. Available at http://dx.doi. org/10.21037/atm-20-7024

Data Sharing Statement: Available at http://dx.doi. org/10.21037/atm-20-7024

Conflicts of Interest: All authors have completed the ICMJE uniform disclosure form (available at http://dx.doi. org/10.21037/atm-20-7024). The authors have no conflicts of interest to declare.

Ethical Statement: The authors are accountable for all aspects of the work in ensuring that questions related to the accuracy or integrity of any part of the work are appropriately investigated and resolved. Experiments were performed under a project license (NO.: EC20200415) granted by institutional ethics committee board of Guangdong Academy of Medical Sciences, in compliance with the National Institutes of Health Guide concerning the Care and Use of Laboratory Animals.

Open Access Statement: This is an Open Access article distributed in accordance with the Creative Commons Attribution-NonCommercial-NoDerivs 4.0 International License (CC BY-NC-ND 4.0), which permits the noncommercial replication and distribution of the article with the strict proviso that no changes or edits are made and the original work is properly cited (including links to both the formal publication through the relevant DOI and the license). See: https://creativecommons.org/licenses/by-nc-nd/4.0/.

\section{References}

1. Fleischmann C, Scherag A, Adhikari NK, et al. Assessment of global incidence and mortality of hospital-treated sepsis. Current estimates and limitations. Am J Respir Crit Care Med 2016;193:259-72.

2. Iwashyna TJ, Ely EW, Smith DM, et al. Long-term cognitive impairment and functional disability among survivors of severe sepsis. JAMA 2010;304:1787-94.

3. Annane D, Sharshar T. Cognitive decline after sepsis. Lancet Respir Med 2015;3:61-9.

4. Smith M, Meyfroidt G. Critical illness: the brain is always in the line of fire. Intensive Care Med 2017;43:870-3.

5. Zhao Z, Nelson AR, Betsholtz C, et al. Establishment and dysfunction of the blood-brain barrier. Cell 2015;163:1064-78.

6. Meneses G, Cárdenas G, Espinosa A, et al. Sepsis: developing new alternatives to reduce neuroinflammation and attenuate brain injury. Ann N Y Acad Sci 2019;1437:43-56.

7. Bosmann M, Russkamp NF, Strobl B, et al. Interruption 
of Macrophage-Derived IL-27(p28) Production by IL10 during Sepsis Requires STAT3 but Not SOCS3. J Immunol 2014;193:5668-77.

8. Guo W, Liu W, Chen G, et al. Water-soluble andrographolide sulfonate exerts anti-sepsis action in mice through down-regulating p38 MAPK, STAT3 and NF-кB pathways. Int Immunopharmacol 2012;14:613-9.

9. Cai B, Cai JP, Luo YL, et al. The specific roles of JAK/STAT signaling pathway in sepsis. Inflammation 2015;38:1599-608.

10. Mukherjee S, Allen RM, Lukacs NW, et al. STAT3Mediated IL-17 Production by Postseptic T Cells Exacerbates Viral Immunopathology of the Lung. Shock 2012;38:515-23.

11. DeMartini T, Nowell M, James J, et al. High fat dietinduced obesity increases myocardial injury and alters cardiac STAT3 signaling in mice after polymicrobial sepsis. Biochim Biophys Acta Mol Basis Dis 2017;1863:2654-60.

12. Chang X, Hu L, Ma X, et al. Influence of roflumilast on sepsis mice through the JAK/STAT signaling pathway. Eur Rev Med Pharmacol Sci 2019;23:1335-41.

13. Liu MJ, Shengying B, R. NJ, et al. Zinc Regulates the Acute Phase Response and Serum Amyloid A Production in Response to Sepsis through JAK-STAT3 Signaling. Plos One 2014;9:e94934.

14. Yuan FH, Chen YL, Zhao Y, et al. microRNA-30a inhibits the liver cell proliferation and promotes cell apoptosis through the JAK/STAT signaling pathway by targeting SOCS-1 in rats with sepsis. J Cell Physiol 2019;234:17839-53.

15. Lv X, Zhang Y, Cui Y, et al. Inhibition of microRNA-155 relieves sepsis-induced liver injury through inactivating the JAK/STAT pathway. Mol Med Rep 2015;12:6013-8.

16. Timofeeva OA, Chasovskikh S, Lonskaya I, et al. Mechanisms of unphosphorylated STAT3 transcription factor binding to DNA. J Biol Chem 2012;287:14192-200.

17. Darnell JE, Kerr IM, Stark GR. Jak-STAT pathways and transcriptional activation in response to IFNs and other extracellular signaling proteins. Science 1994;264:1415-21.

18. Guo L, Chen C, Shi M, et al. Stat3-coordinated Lin-28let-7-HMGA2 and miR-200-ZEB1 circuits initiate and maintain oncostatin M-driven epithelial-mesenchymal transition. Oncogene 2013;32:5272-82.

19. Cai Y, Chen H, Mo X, et al. Toxoplasma gondii inhibits apoptosis via a novel STAT3-miR-17-92-Bim pathway in macrophages. Cell Signal 2014;26:1204-12.

20. Dan C, Jinjun B, Zi-Chun H, et al. Modulation of TNF- $\alpha$ mRNA stability by human antigen $\mathrm{R}$ and miR181s in sepsis-induced immunoparalysis. EMBO Mol Med 2014;7:140-57.

21. Sun X, Icli B, Wara AK, et al. MicroRNA-181b regulates $\mathrm{NF}-\kappa \mathrm{B}-$ mediated vascular inflammation. J Clin Invest 2012;122:1973-90.

22. Mcclure C, McPeak MB, Youssef D, et al. Stat3 and C/ EBP $\beta$ synergize to induce miR-21 and miR-181b expression during sepsis. Immunol Cell Biol 2017;95:42-55.

23. Mcclure C, Brudecki L, Ferguson DA, et al. MicroRNA 21 (miR-21) and miR-181b Couple with NFI-A To Generate Myeloid-Derived Suppressor Cells and Promote Immunosuppression in Late Sepsis. Infect Immun 2014;82:3816-25.

24. Alkhateeb T, Kumbhare A, Bah I, et al. S100A9 maintains myeloid-derived suppressor cells in chronic sepsis by inducing miR-21 and miR-181b. Mol Immunol 2019;112:72-81.

25. Lu Y, Xu X, Dong R, et al. MicroRNA-181b-5p attenuates early postoperative cognitive dysfunction by suppressing hippocampal neuroinflammation in mice. Cytokine 2019;120:41-53.

26. Seymour CW, Liu VX, Iwashyna TJ, et al. Assessment of clinical criteria for sepsis: for the Third International Consensus Definitions for Sepsis and Septic Shock (Sepsis-3). JAMA 2016;315:762-74.

27. Guo R, Fan G, Zhang J, et al. A 9-microRNA Signature in Serum Serves as a Noninvasive Biomarker in Early Diagnosis of Alzheimer's Disease. J Alzheimers Dis 2017;60:1365-77.

28. Ding H, Huang Z, Chen M, et al. Identification of a panel of five serum miRNAs as a biomarker for Parkinson's disease. Parkinsonism Relat Disord 2016;22:68-73.

29. Waller R, Goodall EF, Milo M, et al. Serum miRNAs miR-206, 143-3p and 374b-5p as potential biomarkers for amyotrophic lateral sclerosis (ALS). Neurobiol Aging 2017;55:123-31.

30. Jamal Uddin M, Joe Y, Kim SK, et al. IRG1 induced by heme oxygenase-1/carbon monoxide inhibits LPSmediated sepsis and pro-inflammatory cytokine production. Cell Mol Immunol 2016;13:170-9.

31. Bavunoglu I, Genc H, Konukoglu D, et al. Oxidative stress parameters and inflammatory and immune mediators as markers of the severity of sepsis. J Infect Dev Ctries 2016;10:1045-52.

32. Zhang $\mathrm{W}$, He J. Interleukin-6 is a key factor for immunoglobulin-like transcript-4-mediated immune injury in sepsis. J Intensive Care 2018;6:22 .

33. Baggiolini M, Clark-Lewis I. Interleukin-8, a chemotactic 
and inflammatory cytokine. FEBS Lett 1992;307:97-101.

34. Kuang L, Zhu Y, Zhang J, et al. A novel cross-linked haemoglobin-based oxygen carrier is beneficial to sepsis in rats. Artif Cells Nanomed Biotechnol 2019;47:1496-504.

35. Li X, Tian F, Wang F. Rheumatoid arthritis-associated microRNA-155 targets SOCS1 and upregulates TNF- $\alpha$ and IL-1 $\beta$ in PBMCs. Int J Mol Sci 2013;14:23910-21.

36. Cao YZ, Tu YY, Chen X, et al. Protective effect of Ulinastatin against murine models of sepsis: inhibition of TNF- $\alpha$ and IL- 6 and augmentation of IL-10 and IL-13. Exp Toxicol Pathol 2012;64:543-7.

37. Halytskiy V. Immune cells can cause inappropriate inflammation leading to the miRNAome shift and cell transformation. Ann Oncol 2016;27:viii7.

38. Tang Y, Soroush F, Sun S, et al. Protein kinase C-delta inhibition protects blood-brain barrier from sepsis-induced vascular damage. J Neuroinflammation 2018;15:309.

39. Margotti W, Giustina AD, de Souza Goldim MP, et al. Aging influences in the blood-brain barrier permeability and cerebral oxidative stress in sepsis. Exp Gerontol 2020;140:111063.

40. Huang ZS, Xie DQ, Xu LJ, et al. Tetramethylpyrazine

Cite this article as: Chen SL, Cai GX, Ding HG, Liu XQ, Wang ZH, Jing YW, Han YL, Jiang WQ, Wen MY. microRNA-181b promoted blood-brain barrier impairment through the JAK/STAT3 signaling pathway by targeting sphingosine-1-phosphate receptor 1 in septic rats. Ann Transl Med 2020;8(21):1458. doi: 10.21037/atm-20-7024
Ameliorates Lipopolysaccharide-Induced Sepsis in Rats via Protecting Blood-Brain Barrier, Impairing Inflammation and Nitrous Oxide Systems. Front Pharmacol 2020;11:562084.

41. Feng A, Rice AD, Zhang Y, et al. S1PR1-associated molecular signature predicts survival in patients with sepsis. Shock 2020;53:284-92.

42. Gaengel K, Niaudet C, Hagikura K, et al. The sphingosine-1-phosphate receptor S1PR1 restricts sprouting angiogenesis by regulating the interplay between VE-cadherin and VEGFR2. Dev Cell 2012;23:587-99.

43. Viviano J, Krishnan A, Wu H, et al. Electrophoretic Mobility Shift in Native Gels Indicates Calciumdependent Structural Changes of Neuronal Calcium Sensor Proteins. Analytical Biochemistry 2015. doi: 10.1016/j.ab.2015.11.005.

44. Dalvi P, Sun B, Tang N, et al. Immune activated monocyte exosomes alter microRNAs in brain endothelial cells and initiate an inflammatory response through the TLR4/ MyD88 pathway. Sci Rep 2017;7:9954.

(English Language Editors: J. Teoh and J. Reynolds) 